\title{
A dual-mass capacitive-readout accelerometer operated near pull-in
}

\author{
P. Woestyn*, J. Raman*, P. Rombouts*, L. Weyten* and P. De Baets ${ }^{\dagger}$ \\ ${ }^{*}$ Electronics and Information Systems $/{ }^{\dagger}$ Mechanical Construction and Production, Ghent University \\ Email: pierre.woestyn@ugent.be
}

\begin{abstract}
A mechanical two-mass configuration and a readout circuit for a single-axis capacitive-readout accelerometer with $\Sigma \Delta$ force-feedback is presented. The system reduces electrical and quantisation input-referred noise through the use of negative springs, reduced gaps in the readout capacitors and maximised readout voltage. A theoretical analysis and simulation results are discussed.
\end{abstract}

\section{INTRODUCTION}

A typical micromechanical accerometer consists of a proof mass suspended with springs. The force-to-displacement transfer function is commonly modelled as a second-order system described by

$$
M(s)=\frac{x(s)}{F(s)}=\frac{1}{m s^{2}+b s+k_{m}}
$$

with $m, b$ and $k_{m}$ the mass, damping coefficient and mechanical spring constant respectively. To convert the resulting displacement to a voltage, capacitive readout using parallelplate capacitors is frequently used (figure 1a). In this case, the electronic amplifier responsible for the conversion is often a significant source of noise [1].

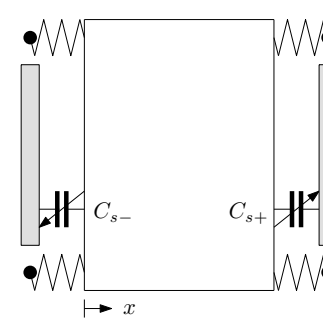

(a)

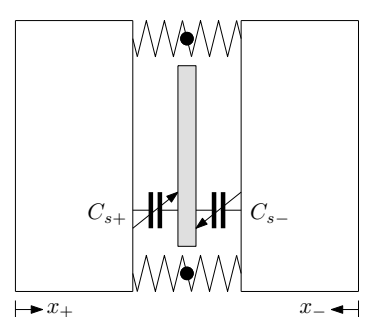

(b)
Figure 1. Simplified representation of a single-axis accelerometer with parallel-plate capacitive readout: (a) conventional solution with a single proof mass and two stator electrodes; (b) proposed solution with two masses and a single stator.

The impact of this noise can be reduced by increasing the sensitivity of the mechanical structure and readout. Three methods can be discerned. Reducing the capacitor gap increases position-to-capacitance-change gain [2]. A second approach is to increase the excitation voltage used in the readout circuit [1] (see section III-B for a detailed discussion). Increasing the sensitivity of the mechanical part also reduces the input-referred noise [3]. A straightforward way to do this is to decrease $k_{m}$. However, robustness suffers as shocks more readily damage the device.
We propose a system that adds negative springs (see section II), thus increasing sensitivity without sacrificing robustness. At the same time, the readout capacitor gap is reduced during operation and the highest excitation voltage possible is used in the readout circuit.

\section{NEGATIVE SPRINGS}

Consider the capacitors between stators and proof mass of figure 1a. It is well known that a voltage $V$ across each stator and proof mass results in an attractive force between them, given by

$$
F_{P P}=\frac{C_{0} x_{0} V^{2}}{2\left(x_{0}-x\right)}
$$

with $x_{0}$ and $C_{0}$ the gap and capacitance at rest, and $x$ the displacement. This force increases as the gap narrows, and can thus be considered a negative spring. The net result is that the mechanical spring constant is partially canceled. The magnitude of the spring constant $k_{e}$ also increases with a reduced gap. It can be shown that a stable system is only obtained for $V<V_{P I}=\sqrt{k_{m} x_{0}^{2} / C_{0}}$, with $V_{P I}$ the pull-in voltage [4], [5]. Beyond this point, the mechanical spring is overpowered and the proof mass is drawn to one of the stators (pull-in).

These springs can be used to cancel $k_{m}$ to increase the mechanical sensitivity [3]. This concept is also used in this work, although compared to prior work [3] a higher excitation voltage is used, and the readout capacitor gap is reduced.

\section{PROPOSED SYSTEM}

We propose a system composed of a dual-mass mechanical structure (section III-A), a novel readout circuit (III-B) and two control loops to maintain the proof masses at the desired position (III-C-III-D).

\section{A. Mechanical part}

By reducing the gap between the readout capacitor plates, the sensitivity can be increased [2]. However, excessively small gaps will reduce yield through fabrication problems with etching and stiction.

Another approach is to decrease the gap during operation. In a conventional differential accelerometer this is not easy to achieve. One method, displacement of the proof mass, will introduce asymmetry and is thus to be avoided. On the other hand, various ways to move the normally fixed stators have 
been proposed [2], but they involve fairly complex mechanical structures which occupy significant die area.

We propose the mechanical configuration depicted in figure $1 \mathrm{~b}$. Two independent, nominally equal proof masses are employed with inverse sensitivity to acceleration along the $x$ axis. Each mass is now half as large as the single proof mass of an equivalent ordinary accelerometer. Calling the displacement of the left (right) proof mass $x_{+}\left(x_{-}\right)$, a normalised differential and common-mode displacement can be defined as $\hat{x}_{d m}=\left(x_{+}-x_{-}\right) /\left(2 x_{0}\right)$ resp. $\hat{x}_{c m}=\left(x_{+}+x_{-}\right) /\left(2 x_{0}\right)$. The capacitor gap can now be decreased without disturbing the symmetry by increasing $\hat{x}_{c m}$ (see section III-D) while keeping $\hat{x}_{d m} \approx 0$ (III-C).

The sense capacitances $C_{s \pm}$ can then be written as

$$
C_{s \pm}=\frac{C_{0}}{1-\frac{x_{ \pm}}{x_{0}}}=\frac{C_{0}}{1-\left(\hat{x}_{c m} \pm \hat{x}_{d m}\right)}
$$

Again, each mass is susceptible to pull-in; the critical voltage $V_{P I}$ is now lower as no force from the opposite electrode is present and is calculated as $V_{P I}=\sqrt{8 k_{m} x_{0}^{2} / 27 C_{0}}$ [4]. Note that the pull-in point equals $x_{P I}=1 / 3 x_{0}$, independent of other parameters.

For notational convenience we also introduce

$$
\left\{\begin{array}{l}
C_{c m}=\frac{C_{s+}+C_{s-}}{2}=C_{0} \frac{1-\hat{x}_{c m}}{\left(1-\hat{x}_{c m}\right)^{2}-\hat{x}_{d m}^{2}} \\
C_{d m}=\frac{C_{s+}-C_{s-}}{2}=C_{0} \frac{\hat{x}_{d m}}{\left(1-\hat{x}_{c m}\right)^{2}-\hat{x}_{d m}^{2}}
\end{array}\right.
$$

\section{B. Readout circuit}

A well-known circuit for capacitive readout is shown in figure 2 [1]. A step $\Delta V_{e x}$ applied at the proof mass results in an output voltage proportional to $C_{s+}-C_{s-}$. An input commonmode feedback (ICMFB) amplifier stabilises the commonmode voltage at the stator electrodes.

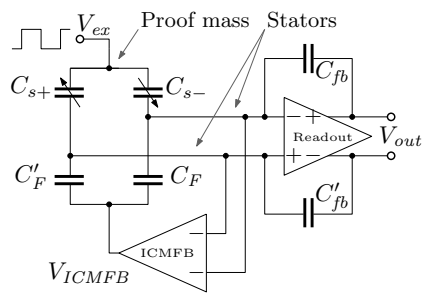

Figure 2. Simplified capacitive readout circuit of [1] (reset and CDS not shown).
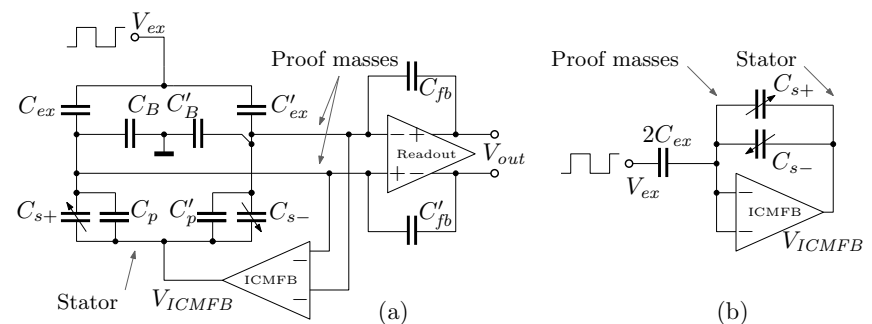

(b)

Figure 3. Proposed readout circuit: (a) complete circuit; (b) equivalent circuit under the assumption $C_{+} \approx C_{-}\left(C_{p}\right.$ and $C_{B}$ omitted). Compare to [5, figure 9].
We propose the readout configuration of figure 3a: fundamentally identical to 2 , save for $C_{e x} / C_{F}$ and $C_{s \pm}$ which have been interchanged. Parasitic capacitors have also been included: $C_{B}$ to ground at each proof mass terminal of $C_{s \pm}$, and $C_{p}$ in parallel with the readout capacitor. A step applied on the excitation capacitors $C_{e x}$ causes an opposite step at the output of the ICMFB amplifier

$$
\Delta V_{I C M F B}=-\frac{C_{e x}}{C_{p}+C_{c m}} \Delta V_{e x}
$$

The change in $V_{I C M F B}$ in turn results in the differential readout

$$
\Delta V_{\text {out }}=-\frac{C_{d m}}{C_{f b}} \Delta V_{I C M F B}+v_{o n}
$$

with $C_{f b}$ the feedback capacitor and $v_{o n}$ the output referred noise of the readout amplifier. Merging equations 3-5 yields

$$
\Delta V_{\text {out }}=\frac{C_{e x}}{C_{f b}} \frac{C_{c m}}{C_{p}+C_{c m}} \frac{\hat{x}_{d m}}{1-\hat{x}_{c m}} \Delta V_{e x}+v_{\text {on }}
$$

$v_{\text {on }}$ can be related to the input-referred noise $v_{i n}$ as

$$
v_{\text {on }}=\frac{C_{c m}+C_{e x}+C_{f b}+C_{p}+C_{B}}{C_{f b}} v_{i n} .
$$

\section{Differential stabilisation}

It is well known that force feedback in an accelerometer increases dynamic range and decreases non-linearity by reducing the displacement of the proof mass. $\Sigma \Delta$ modulation is commonly employed for this purpose [6], [7], giving a direct digital readout.

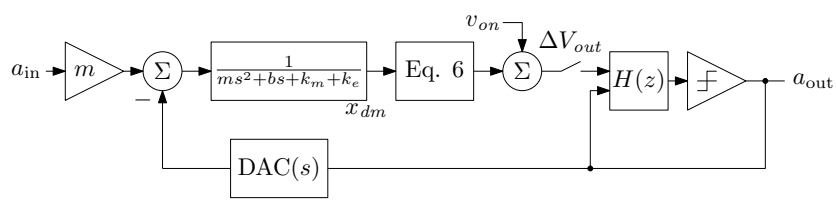

Figure 5. Differential stabilisation part of the full model. A electrical spring $k_{e}<0$ appears in parallel to $k_{m}$.

In our case $\hat{x}_{d m}$ is stabilised by a fourth-order $\Sigma \Delta$ force feedback loop (figures 4-5). The loop uses an unconstrained architecture, negating the requirement of a compensator for stability [8], [9]. Two poles result from $M(s)$; two additional electric integrators from the electrical loop filter $H(z)$ shape the quantisation noise further.

As discussed below, we try to maximise the sensitivity, resulting in increased loop gain. The $\Sigma \Delta$ loop was designed with this in mind.

\section{Common-mode stabilisation}

Given the force feedback, we may assume $\hat{x}_{d m} \approx 0$. Under this condition inspecting equation (3) reveals the commonmode capacitance behaves like a single parallel-plate capacitor. Results derived for the latter (discussed in e.g. [4], [5]) are now applicable for the common-mode behaviour of our system.

It is well known that voltage control of moving parallelplate capacitors results in instability beyond the pull-in point. 


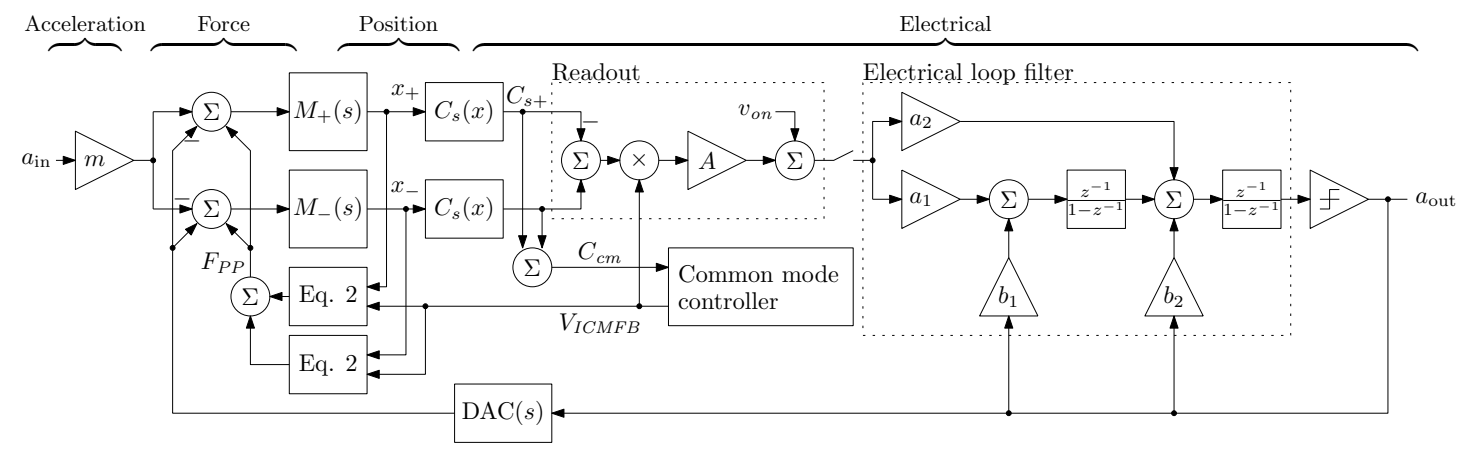

Figure 4. Full system model, with the different signal domains described above. $a_{1}, a_{2}, b_{1}$ and $b_{2}$ are constants. The common mode controller is depicted in more detail in figure 6.

On the other hand, charge control is stable for the full range of displacement if no parasitic capacitance is present [5]. Even with parasitic capacitance charge control always results in a larger range of stable control than voltage control.

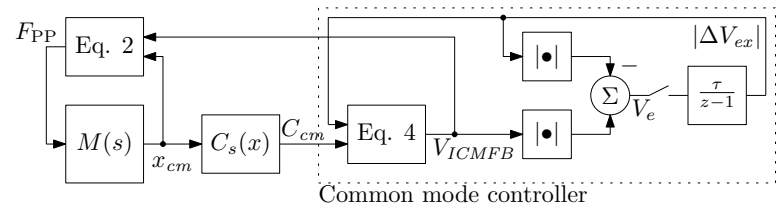

Figure 6. Common-mode stabilisation part of the full model.

The proposed readout circuit uses charge control for the common mode: assuming $C_{+} \approx C_{-}$, both can be considered parallel, and the equivalent single capacitor is located in the feedback path of the ICMFB amplifier (figure $3 b$ ).

We now derive a control loop to stabilise $\hat{x}_{c m}$ (see figures 4 and 6). Still assuming $\hat{x}_{d m} \approx 0$, and neglecting $C_{p}$, equation (4) becomes

$$
\Delta V_{I C M F B}=-\frac{C_{e x}}{C_{0}}\left(1-\hat{x}_{c m}\right) \Delta V_{e x}
$$

Choose $C_{e x}$ now equal to $C_{c m}$ at the desired operating point $\hat{x}_{r e f}$, i.e. $C_{e x}=C_{0} /\left(1-\hat{x}_{r e f}\right)$, and introduce the error signal

$$
\begin{aligned}
V_{e} & =\left|\Delta V_{\text {ICMFB }}\right|-\left|\Delta V_{e x}\right| \\
& =\left|\Delta V_{e x}\right|\left(\frac{1-\hat{x}_{c m}}{1-\hat{x}_{r e f}}-1\right)=\left|\Delta V_{e x}\right| \frac{\hat{x}_{r e f}-\hat{x}_{c m}}{1-\hat{x}_{r e f}}
\end{aligned}
$$

Stable control can then be obtained by integrating $V_{e}$ and using the output as $\Delta V_{e x}$. If $\hat{x}_{c m}$ is too low, $V_{e}$ is positive and $\Delta V_{e x}$ increases, causing $\left|\Delta V_{I C M F B}\right|$ to increase. As the attractive force between the parallel plate grows with $\Delta V_{I C M F B}^{2}, \hat{x}_{c m}$ increases until $V_{e}=0$. In principle any operating point can be obtained; in practice limits are imposed by parasitic capacitances [5] and imperfect differential nulling.

\section{E. Discussion}

In a two-chip solution with the mechanical and electrical chip connected by bond wires the typical parasitic capacitance $C_{p}$ and especially $C_{B}$ is very high-in the same order of magnitude as the sensing capacitance [1]. Under these conditions equations 6-7 show that $C_{f b}$ has little influence on the signal-to-electrical-noise ratio (SENR), while $\Delta V_{e x}$ and $\hat{x}_{c m}$ is preferably as high as possible. For operating points $\hat{x}_{c m}<1 / 3$ an increasing $\hat{x}_{c m}$ results in higher $\Delta V_{e x}$. Beyond this point, $\Delta V_{e x}$ decreases rapidly and stabilising $\hat{x}_{d m}$ becomes difficult. Thus, choosing the operating point $\hat{x}_{c m}=1 / 3$ optimises SENR. At this point the step $\Delta V_{e x}$ equals $2 V_{P I}$, much higher than what is conventionally used in readout circuits, with a commensurate improvement in noise. At the same time the magnitude of the voltage of the parallel plates does not change: only the sign does. Compared to [3] this eliminates periodic changes of the negative springs.

When the operating point is chosen perfectly at pull-in, $k_{m}$ is cancelled by the electrical spring. Thus, the proof mass behaves like a floating mass with a very high sensitivity. Equation (1) now includes a perfect integrator, and lowfrequency quantisation and electrical noise is well suppressed.

\section{VERIFICATION}

Operation of the system was verified by a behavioural simulation based on the model of figure 4. For the simulation, a two-mass accelerometer design was used consisting of two identical $1.1 \times 2 \mathrm{~mm}^{2}$ proof masses of $34.8 \mu \mathrm{g}$ side-by-side. The quality factor is 1.77 while $k_{m}=26.5 \mathrm{~N} / \mathrm{m}$; this gives a resonance frequency of $4.39 \mathrm{kHz}$.

Comb capacitors on each proof mass are used for differential feedback; the maximal force they can exert is $25.6 \mu \mathrm{N}$. Parallel-plate readout capacitors are placed on both sides of the proof mass in a array of $2 \times 132$ fingers yielding $C_{0}=2.63 \mathrm{pF}$. Inevitably, parasitic back capacitors will be present $(1.32 \mathrm{pF}$ in our case), the capacitance of which decreases with increasing $x_{ \pm}$. This effect has been ignored so far, but is included in the simulation. It will decrease the capacitive-readout gain slightly and decrease the pull-in point to $x_{P I} / x_{0} \approx 0.307$.

The sampling frequency is $200 \mathrm{kHz}$ which gives an oversampling ratio of about 46 compared to the natural bandwidth of the proof mass.

In simulation, a very high noise level was chosen for the readout amplifier $(1 \mu \mathrm{V} / \sqrt{\mathrm{Hz}}$ referred to the amplifier input in a noise bandwidth of $100 \mathrm{MHz}$ ) was chosen in order to obtain a clear noise floor. Similar to the quantisation noise transfer 


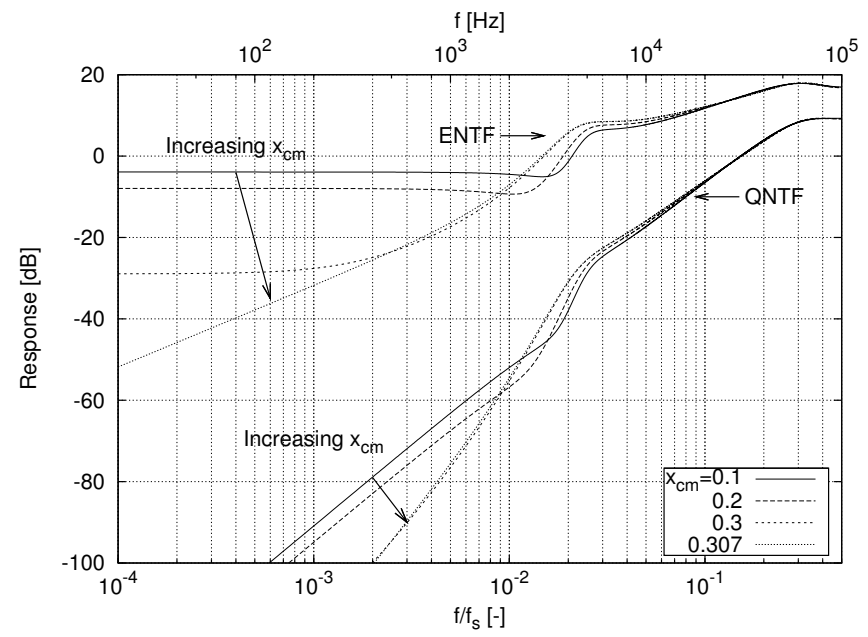

Figure 7. Transfer functions, showing the increasing noise suppression in the signal band for increasing $\hat{x}_{c m}$. For $\hat{x}_{c m}>x_{P I} / x_{0}$, noise suppression worsens again.

function (QNTF), the electrical noise transfer function (ENTF) can be defined as the transfer function of the electrical noise to the output bitstream. Figure 7 shows the QNTF and ENTF for various operating points calculated from the differential signal path of figure 4. It can be seen that the decreasing total spring constant causes the noise transfer levels to fall in the signal band. The slope of the QNTF and ENTF in the signal band changes only when the total spring becomes exactly zero.

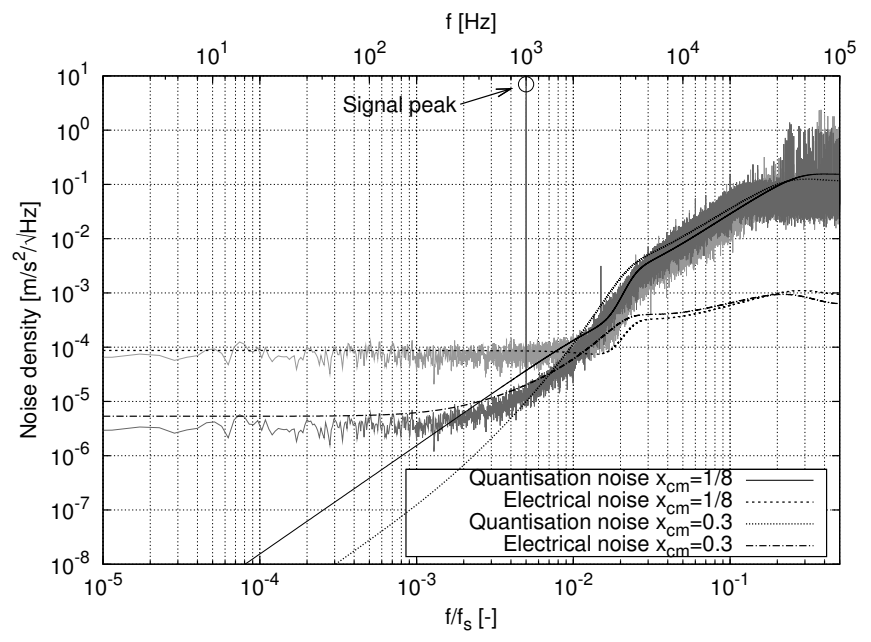

Figure 8. Input-related spectrum of the output for $a_{\text {in }}=20 \mathrm{~m} / \mathrm{s}^{2}$ at $1 \mathrm{kHz}$ (four times averaged), for $\hat{x}_{c m}=1 / 8$ (light grey) and $\hat{x}_{c m}=0.3$ (dark grey). The predicted quantisation and electrical noise levels are also plotted.

The calculated transfer functions were verified in simulation. Figure 8 shows the output spectra, referred back to the input, for two operating points. The simulated noise floor decreases from 78.2 to $3.60 \mu \mathrm{m} / \mathrm{s}^{2} / \sqrt{\mathrm{Hz}}$ as $\hat{x}_{c m}$ is changed from $1 / 8$ to 0.3 . Figure 9 shows, as a function of the operating point, the noise level averaged over $0-100 \mathrm{~Hz}$ and the maximal input amplitude for which the system is stable. The predicted and simulated noise levels show reasonable agreement for

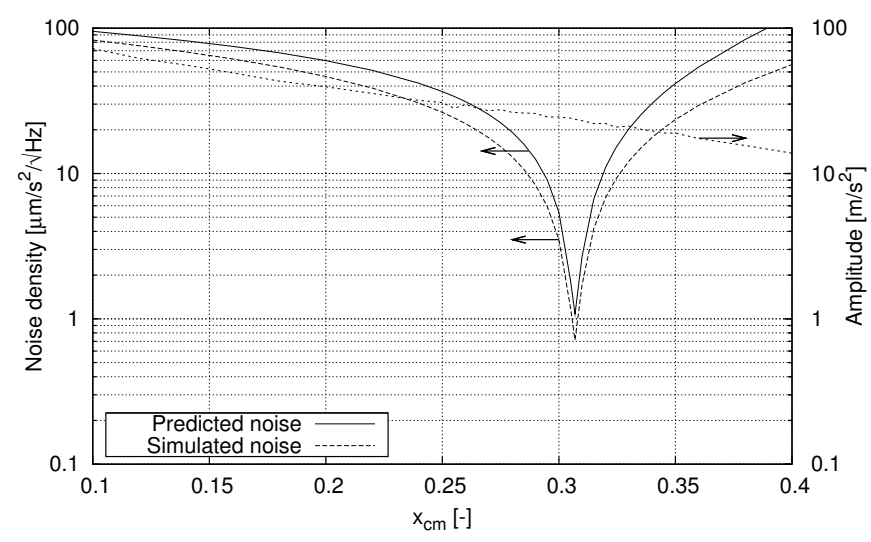

Figure 9. Maximal input amplitude (right scale, at a frequency of $1 \mathrm{kHz}$ ) and average noise density below $100 \mathrm{~Hz}$ as a function of $\hat{x}_{\mathrm{cm}}$.

low $\hat{x}_{c m}$.

\section{CONCLusions}

A dual-mass accelerometer system with parallel-plate capacitors readout and a novel readout circuit allows operation on the pull-in point with a reduced capacitor gap and maximal excitation amplitude. These three factors all improve quantisation and electrical noise. Behavioural simulations of the system show a reasonable agreement with predicted noise levels.

The readout circuit is equally applicable to single-mass accelerometers or accelerometers not operated near pull-in. For these applications too, the excitation step is the highest possible for a given tolerated negative spring effect, with corresponding benefits with respect to noise.

\section{REFERENCES}

[1] M. Lemkin and B. Boser, "A three-axis micromachined accelerometer with a CMOS position-sense interface and digital offset-trim electronics," IEEE Journal of solid-state circuits, vol. 34, pp. 456-468, Apr 1999.

[2] C. Acar and A. Shkel, "Post-release capacitance enhancement in micromachined devices," in Proceedings of the IEEE Sensors 2004, Vols 1-3, pp. 268-271, IEEE, 2004. Vienna, Austria.

[3] M. Handtmann, R. Aigner, A. Meckes, and G. Wachutka, "Sensitivity enhancement of MEMS inertial sensors using negative springs and active control," Sensors and actuators A-Physical, vol. 97-8, pp. 153-160, Apr 12002 .

[4] G. Nielson and G. Barbastathis, "Dynamic pull-in of parallel-plate and torsional electrostatic mems actuators," Journal of Microelectromechanical Systems, vol. 15, pp. 811-821, Aug 2006.

[5] J. Seeger and B. Boser, "Charge control of parallel-plate, electrostatic actuators and the tip-in instability," Microelectromechanical Systems, Journal of, vol. 12, pp. 656-671, Oct 2003.

[6] V. Petkov and B. Boser, "A fourth-order Sigma Delta interface for micromachined inertial sensors," IEEE Journal of solid-state circuits, vol. 40, pp. 1602-1609, Aug 2005.

[7] Y. Dong, M. Kraft, C. Gollasch, and W. Redman-White, "A highperformance accelerometer with a fifth-order sigma-delta modulator," Journal of micromechanics and microengineering, vol. 15, pp. S22-S29, Jul 2005.

[8] J. Raman, P. Rombouts, and L. Weyten, "An unconstrained architecture for systematic design of higher order sigma delta force-feedback loops," IEEE Transactions on Circuits and Systems I - Regular Papers, vol. 55, pp. 1601-1614, 72008.

[9] J. Raman, E. Cretu, P. Rombouts, and L. Weyten, "A Closed-Loop Digitally Controlled MEMS Gyroscope With Unconstrained Sigma-Delta Force-Feedback," IEEE Sensors journal, vol. 9, pp. 297-305, Mar 2009. 